\title{
Trojan planets in HD 108874? (Research Note)
}

\author{
R. Schwarz ${ }^{2}$, R. Dvorak ${ }^{1}$, E. Pilat Lohinger ${ }^{1}$, Á. Süli ${ }^{2}$, and B. Érdi ${ }^{2}$ \\ 1 Institute for Astronomy, University of Vienna, Türkenschanzstr. 17, 1180 Wien, Austria \\ e-mail: dvorak@astro.univie.ac.at \\ 2 Eötvös Loránd University, Departement of Astronomy, Pázmány Péter sétány 1/A, Budapest 1518, Hungary
}

Received 22 August 2006 / Accepted 16 October 2006

ABSTRACT

\begin{abstract}
Aims. It turned out recently that, in addition to a large planet with a semimajor axis $a \sim 1 \mathrm{AU}$ and a low eccentricity $(e \sim 0.07)$, the extrasolar planetary system HD 108874 harbors another massive planet with $2.43 \mathrm{AU}<a<2.93 \mathrm{AU}$. The inner planet is orbiting the G5 host star in the habitable zone $(=\mathrm{HZ})$; so that we could established stable regions for Earth-like Trojan planets.

Methods. We integrated some $10^{5}$ orbits of fictitious Trojans around the Lagrangian points for up to $10^{7}$ years and checked the stability of the orbital elements and their chaoticity with the aid of the Fast Lyapunov Indicator.

Results. It turns out that this multiplanetary system is the first one where - with the uncertainties in eccentricity and semimajor axes of the outer planet - the existence of Trojan terrestrial planets in stable orbits in the HZ is possible for some combinations of the orbital parameters.
\end{abstract}

Key words. celestial mechanics - chaos - stars: planetary systems

\section{Introduction}

Today there are about 209 confirmed planets in 169 extrasolar planetary systems (=EPS). We only have observational evidence for planets from 6 earth masses (Gliese $876 \mathrm{~d}$ ) up to several Jupiter masses. To ensure that an orbit of a planet with a mass comparable to the Earth is stable in the so-called habitable zone $(=\mathrm{HZ})^{1}$ around its host star in planetary systems with Jupiter-like planets, there are different possible configurations:

- a hot Jupiter moves very close to the central star;

- a large planet orbits far enough outside the HZ that it does not significantly perturb the motion of a terrestrial planet inside the $\mathrm{HZ}$;

- if Jupiter itself moves inside the HZ, a terrestrial planet may be in the so-called coorbital motion like a satellite or a Trojan ${ }^{2}$.

A lot of work has already been devoted to the existence of terrestrial planets in EPS during the past ten years. We mention the very interesting work of Menou \& Tabachnik (2003), Laughlin \& Chambers (2002), Pilat-Lohinger \& Dvorak (2002), Pilat Lohinger et al. (2003), and Jones \& Sleep (2002).

${ }^{1}$ Roughly speaking this is the zone where water can be in a liquid state, Kasting (1993). The problem of habitable regions around a host star, which is still somewhat in contradiction, will not be discussed. It depends on the dynamical parameters of the orbits of a planet there, but also on the spectral type and the age of the host star (e.g. Lammer et al. 2003).

2 Trojans are two groups of asteroids that move close to the equilateral equilibrium points (Lagrange points $L_{4}$ and $L_{5}$ ) formed by the Sun, Jupiter and the asteroid. Consequently the Jupiter Trojans are moving either close to $60^{\circ}$ ahead or $60^{\circ}$ behind Jupiter with the same semimajor axis as the planet.
Additional studies concern specific systems e.g. Pál \& Sándor (2003), Dvorak et al. (2003a,b), and Asghari et al. (2004).

In this new work we concentrate on one specific system with two planets and on how terrestrial planets may survive in the 1:1 resonance (Nauenberg 2002). According to Laughlin \& Chambers (2002), a possible formation of two planets in a $1: 1$ mean motion resonance could be the result of an interaction of the forming planets with the protoplanetary disc.

\section{Description of the system}

The chances for a single planetary system having a terrestrial planet moving in the $\mathrm{HZ}$ is a lot greater than for multiplanetary systems. When we check Table 1, it is evident that some of these systems could in fact have such Earth-like satellites and/or Earth-like Trojans (=ET; see e.g. Dvorak et al. 2004; Érdi \& Sándor 2005; Schwarz et al. 2005). Note that in Table 1 (also Table 2) the location and the size of the HZ depends on the spectral type of the the host stars. Main parameters: 1st column: name, 2nd column: spectral type, 3rd column: mass of the star, 4th column: the minimum mass of the giant planet, 5th column: distance (semimajor axis $a$ [AU]) from the central star, 6th column: initial eccentricity of the extrasolar planet, 7 th column: extension of the $\mathrm{HZ}$ [AU], and 8th column: partly inside the $\mathrm{HZ}$ in $[\%]$.

In this study we are interested in a multiple system where one giant planet is moving in the $\mathrm{HZ}$ and another large planet disturbs the motion of the first planet and thus reduces the largeness of stable regions for ETs. Unfortunately, up to now no stable region around the Lagrangian points for any of the multiple planetary systems has been confirmed by theoretical studies (Table 2). Using the results of recent observations, we have one very good candidate, HD 108874 (Vogt et al. 2002). This sunlike 
Table 1. List of all EPSs with only one giant planet moving in the HZ of their host stars.

\begin{tabular}{llllllll}
\hline \hline & & Mass & Mass & $a$ & & HZ & $\begin{array}{l}\text { Partly } \\
\text { in HZ [\%] }\end{array}$ \\
Name & Spec. & {$\left[M_{\text {sol }}\right]$} & {$\left[M_{\text {jup }}\right]$} & {$[\mathrm{AU}]$} & $e$ & {$[\mathrm{AU}]$} & 0.48 \\
HD 93083 & K3V & 0.70 & 0.37 & 0.14 & $0.40-1.30$ & 100 \\
HD 134987 & G5V & 1.05 & 1.58 & 0.78 & 0.24 & $0.75-1.40$ & 58 \\
HD 17051 & G0V & 1.03 & 1.94 & 0.91 & 0.24 & $0.70-1.30$ & 100 \\
HD 28185 & G5 & 0.99 & 5.7 & 1.03 & 0.07 & $0.70-1.30$ & 100 \\
HD 27442 & K2IVa & 1.20 & 1.28 & 1.18 & 0.07 & $0.93-1.80$ & 100 \\
HD 188015 & G5IV & 1.08 & 1.26 & 1.19 & 0.15 & $0.70-1.60$ & 100 \\
HD 114783 & K0 & 0.92 & 0.99 & 1.20 & 0.10 & $0.65-1.25$ & 50 \\
HD 20367 & G0 & 1.05 & 1.07 & 1.25 & 0.23 & $0.75-1.40$ & 76 \\
HD 23079 & (F8)/G0V & 1.10 & 2.61 & 1.65 & 0.10 & $0.85-1.60$ & 35 \\
\hline
\end{tabular}

Table 2. As in Table 1 for multiplanetary systems.

\begin{tabular}{llllllll}
\hline \hline & & Mass & Mass & $a$ & & HZ & Partly \\
Name & Spec. & {$\left[M_{\text {sol }}\right]$} & {$\left[M_{\text {jup }}\right]$} & {$[\mathrm{AU}]$} & $e$ & {$[\mathrm{AU}]$} & in HZ [\%] \\
\hline Gliese 876c & M4V & 0.32 & 0.56 & 0.13 & 0.12 & $0.10-0.20$ & 100 \\
Gliese 876b & M4V & 0.32 & 1.89 & 0.21 & 0.27 & $0.10-0.20$ & 0 \\
HD 82943c & G0 & 1.05 & 0.88 & 0.73 & 0.54 & $0.75-1.40$ & 68 \\
HD 82943b & G0 & 1.05 & 1.63 & 1.16 & 0.41 & $0.75-1.40$ & 0 \\
HD 12661b & G6V & 1.07 & 2.30 & 0.83 & 0.35 & $0.80-1.45$ & 60 \\
HD 12661c & G6V & 1.07 & 1.57 & 2.56 & 0.20 & $0.80-1.45$ & 0 \\
HD 160691b & G3IV-V & 1.08 & 1.67 & 1.50 & 0.31 & $0.85-1.60$ & 44 \\
HD 160691c & G3IV-V & 1.08 & 3.10 & 4.17 & 0.57 & $0.85-1.60$ & 0 \\
HD 160691d & G3IV-V & 1.08 & 0.045 & 0.09 & 0.00 & $0.85-1.60$ & 0 \\
\hline HD 108874b & G5 & 1.0 & 1.36 & 1.051 & 0.07 & $0.7-1.3$ & 100 \\
HD 108874c & G5 & 1.0 & 1.018 & 2.68 & 0.25 & $0.7-1.3$ & 0 \\
\hline
\end{tabular}

star was known to have only one massive planet with approximately Jupitermass and an orbit with low eccentricity in the HZ. But recently a second planet was observed outside the orbit of the already known one (Vogt et al. 2005), which we present in Table 3.

We checked orbits of fictitious bodies in the regions around the Lagrangian points $L_{4}$ and $L_{5}$ via numerical simulations for a wide range of orbital parameters of the second perturbing planet. Our research concentrated on investigations of orbits around $L_{4}$, because test computations showed no significant differences in the stable regions around both equilibrium points.

\section{The dynamical models and the numerical setup}

The dynamical model was a restricted $n$-body problem, where we took the central star, the two large planets, and fictitious Trojans into account. The Trojan planets were thought to have low masses compared to the large planets and were therefore set to $m_{4}=0$. This assumption is reasonable as the results of former computations have shown (Schwarz 2005; Schwarz et al. 2005).

To take the possible observational errors into account, especially of the semimajor axis and the eccentricity of the perturbing outer giant, we computed orbits of fictitious Trojan planets in 9 different models. We took the nominal values (Extrasolar Planets Catalogue maintained by J. Schneider ${ }^{3}$ ), but also lower and upper bounds for both parameters, i.e. eccentricity $e$ and semimajor axis $a$ according to the given error bars (see Table 3 ):

- Models M1x, namely M11, M12, and M13 with $a=2.43 \mathrm{AU}$ and three values for the eccentricity $e=0.18,0.25,0.32$.

- Models M2x, namely M21, M22, and M23 with $a=2.68$ AU and again $e=0.18,0.25,0.32$.

\footnotetext{
${ }^{3}$ http://exoplanet.eu/
}

Table 3. Orbital elements of the EPS HD 108874.

\begin{tabular}{ccc}
\hline \hline NAME & HD 108874 b & HD 108874 c \\
\hline Mass $\left[M_{\mathrm{j}}\right]$ & $1.36( \pm 0.13)$ & $1.018( \pm 0.03)$ \\
$a[\mathrm{AU}]$ & $1.051( \pm 0.02)$ & $2.68( \pm 0.25)$ \\
orbital period [days] & $395.4( \pm 2.5)$ & $1605.8( \pm 88)$ \\
eccentricity & $0.07( \pm 0.04)$ & $0.25( \pm 0.07)$ \\
omega [deg] & $248.4( \pm 36)$ & $17.3( \pm 23)$ \\
\hline
\end{tabular}

- Models M3x, namely M31, M32, and M33 with $a=2.93$ AU and $e=0.18,0.25,0.32$.

For a multiplanetary system it is necessary to integrate long enough to see how the secular resonances between the two large planets (like in the Jupiter - Saturn system) act on the orbit of a Trojan planet. We thus have undertaken two different runs for each model:

1. a complete survey of the region around the Lagrange point with a grid in the argument of perihelion $\Delta \omega=1^{\circ}$ and $\Delta a=$ $0.001 \mathrm{AU}$ for $10^{6}$ years within the borders $270^{\circ}<\omega<360^{\circ}$ and $1.01 \mathrm{AU}<a<1.09 \mathrm{AU}$;

2. two cuts within this region where we fixed, on the one hand, the semimajor axis $a$ (exactly the one of the Lagrange point) and varied the angle $\omega$; on the other hand, we fixed the angle $\omega$ and varied the semimajor axes $a$; the integration time was set to $10^{7}$ years.

Our stability criterion was based on the maximum eccentricity achieved during the integration time; when $e_{\max }>0.3$, it turned out - according to numerical results - that the fictitious body has left the region around the Lagrangian equilibrium point $L_{4}$.

The importance of long-term integrations is evident when we compare the results for different time scales of integration up to $10^{7}$ years (Fig. 7). The stability regions shrink significantly and, 


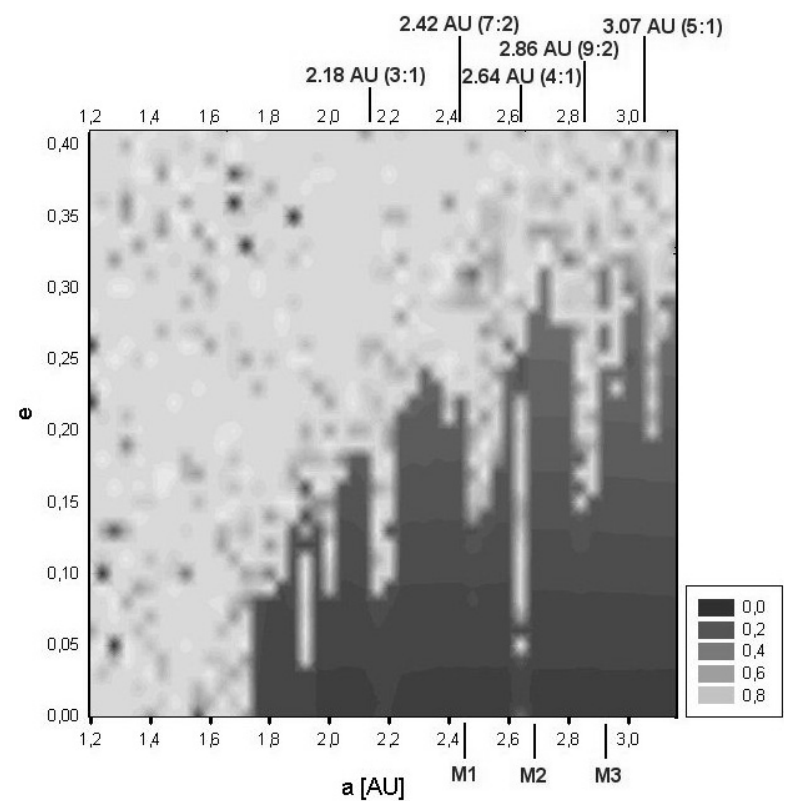

Fig. 1. Stability of the Lagrange point $L_{4}$ taking the outer planet's perturbations into account. On the $x$-axis we plotted the initial semimajor axes, on the $y$-axis the initial eccentricity of the second planet; light grey indicates unstable motions, and dark grey indicates motion of a stable Trojan, where we used $e_{\max }$ during the whole integration as a check.

for the long integration time, there is only a small stable region around the equilibrium point.

Finally we mention that the integration method was the Lieintegration ${ }^{4}$ already used extensively in many of our applications (e.g. Hanslmeier \& Dvorak 1984; Lichtenegger 1984; Dvorak et al. 2004). We also used a different method to ensure the numerical results of the computations, namely the fast Lyapunov indicator (FLI, Froeschle et al. 1997). This is a fast method of qualitatively distinguishing between regular and chaotic motion in a dynamical system. This indicator is defined as the norm of the largest tangent vector (see Froeschlé et al. 1997).

\section{The stability of the equilateral equilibrium points}

First of all, we checked the numerical stability of the point $L_{4}$ itself. In the simple elliptic restricted problem, ignoring the outer planet, this equilibrium point is well inside the stable region for all given values of the eccentricity of the planet and the mass ratio of the primary bodies is well below the value $m_{\text {planet }} / m_{\text {star }}<$ $0.04 \ldots$ This is also true in the full three-body problem when the Trojan planet has a mass comparable to the Earth (Marchal 1990; Schwarz et al. 2005). When we take the second planet into account in our model then the dynamics changes significantly. In Fig. 1 we ploted the maximum eccentricity on the $a, e$ plane, where we show the results for integrations of orbits of objects initially started exactly in $L_{4}$ of the inner giant planet for an integration time of $10^{7}$ years. We varied the initial semimajor axis of the outer planet with $\Delta a=0.02 \mathrm{AU}$ and the initial eccentricity with $\Delta e=0.01$ in the borders covering the uncertainties well in the determined orbital elements of the second planet.

In the respective plot (Fig. 1) we also marked the positions of the mean motion resonances (=MMR) between the two large planets. From this plot we can see that the model M1x is quite

${ }^{4}$ A high order integration method with an automatic step-size control.

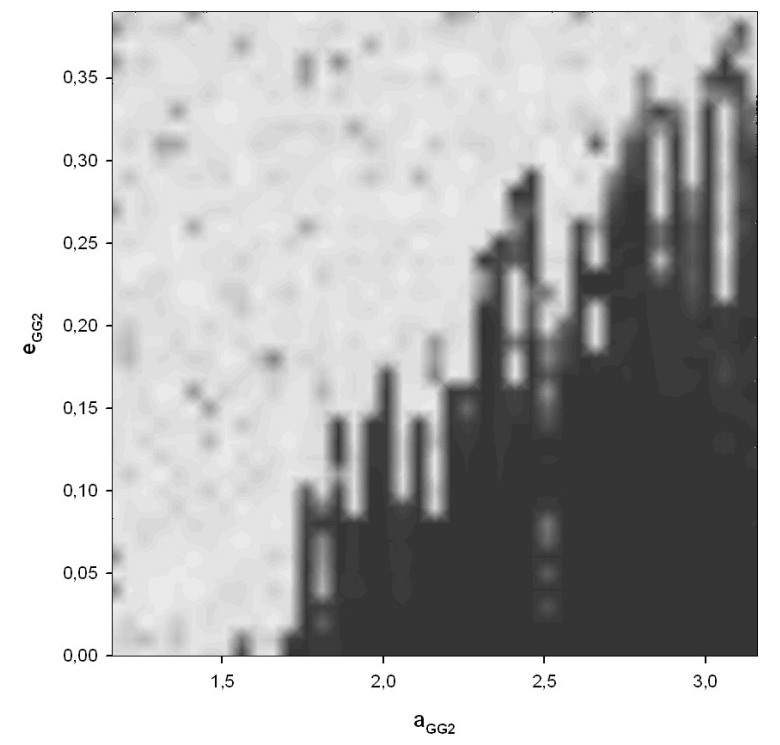

Fig. 2. Captions similar to Fig.1 but with results from the computations of the FLIs. GG2 stands for the outer Gasgiant.

Stability of the 2 planets

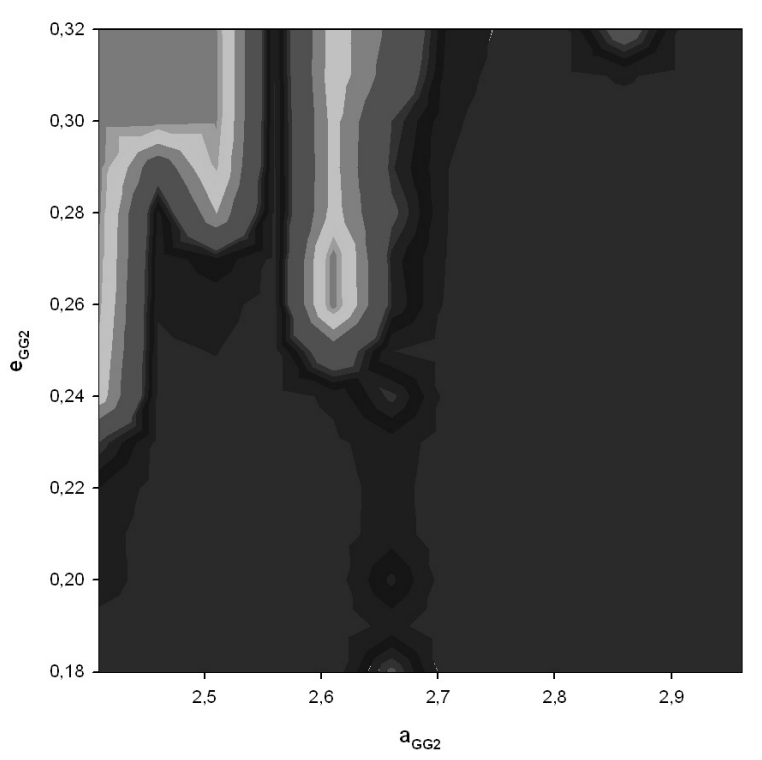

Fig. 3. FLI for different initial conditions of the outer planet in $a$ ( $x$-axis) and $e$ ( $y$-axis). In the upper left corner the two giant planets are in a weak chaotic zone, and the large region in dark grey corresponds to regular orbits of both planets.

close to the 7:2 resonance, that the model $\mathrm{M} 2 \mathrm{x}$ is far from dangerous 4:1 MMR, and that the model M3x is far from the 5:1 MMR. The results of the computations of the FLIs are shown in Fig. 2 and are very similar to the ones of Fig. 1. The vertical stripes of chaotic orbits correspond to MMRs.

We also made computations in the framework of the 3-body problem (the host star and two planets) for different $a$ and $e$ of the outer planet to check the stability of the planets by using the FLIs. It turns out (Fig. 3) that for some of the orbital parameters the two planets are moving in weakly chaotic orbits (but stable up to $10^{8}$ years). In Fig. 4 we show such an orbit with irregular jumps in eccentricity. 


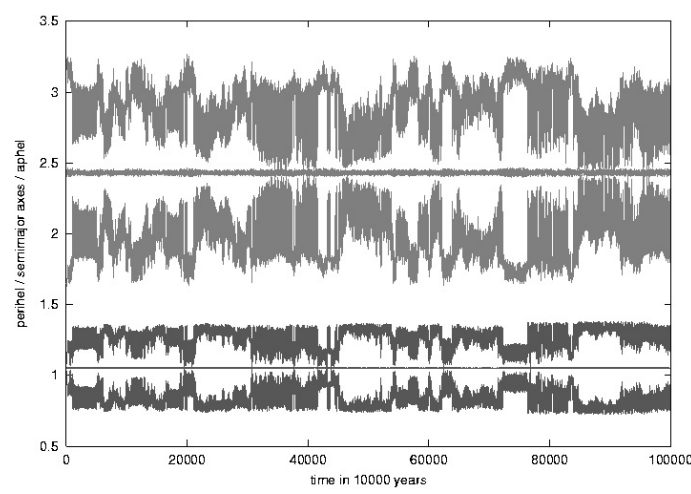

Fig. 4. The chaotic orbits of both planets of HD 108874 with the initial conditions M13 for $10^{8}$ years.

\section{Results of the entire region around $L_{4}$}

It turns out that the model M1x for all three different eccentricities do not have any stable orbits for Trojans around $L_{4}$. For M12 and M13, this is due to the chaoticity of the orbits of the two planets themselves, and M23 is also in such a chaotic domain. The orbits of the fictitious Trojans, for the largest initial eccentricity ( $e=0.32$ ) in models M23 and M33, are also unstable and the Trojan planets do not remain in the vicinity of $L_{4}$ for time scales longer than $1 \mathrm{Myr}$; this is already visible from Figs. 1 and 2. The stable region for the Trojan planets is shown in Fig. 5 (upper graph) for M21, where we can see that this region around the equilibrium point $L_{4}$ (located at $a=1.051$ and $\omega=308^{\circ}$ ) extends from $295^{\circ}<\omega<325^{\circ}$ and $1.035 \mathrm{AU}<a<1.075 \mathrm{AU}$. For M22 (Fig. 5, lower graph) we see only some spots of stable orbits, which we suspected would disappear for a longer integration. In fact the cuts (see next chapter) confirm this behavior that there are not many orbits that are left to be stable ETs. In Fig. 6 (upper graph) for M31 we again observe a slightly larger stable region around the equilibrium point, which extends from $290^{\circ}<\omega<325^{\circ}$ and $1.025 \mathrm{AU}<a<1.08 \mathrm{AU}$. It can be understood that in this model the perturbing planet is farther away from the inner planet and - which is maybe more important also far away from any low order MMR. We show the extension in semimajor axes and $\omega$ in Table 4 for different models. The stable region in model M32 almost disappears for a higher eccentricity of the outer planet. M21* and M31* show the results for more massive planets ${ }^{5}$.

\section{Long-term integration cuts of the stability regions}

As already mentioned in a system with two planets, we need to take into account the secular resonances between the two planets and how they act on the orbit of a Trojan. The effect was recently explained fully by Robutel et al. (2005) for the Jupiter Trojans. Therefore, to define the stability regions with greater accuracy, we need to integrate long enough to include the effects of secular resonances. In Fig. 7 we show how the largeness with respect to the angular distance to $L_{4}$ shrinks with a longer integration-time.

We decided to extend the integration time to $10^{7}$ years for two cuts through $L_{4}$. In one direction we fixed $\omega=\omega_{\text {planet }}+60^{\circ}$ and varied $a$, and vice versa in the other direction, we fixed

\footnotetext{
550 percent larger to take into account the influence of the inclination of the orbits with respect to the line of sight of the observations.
}
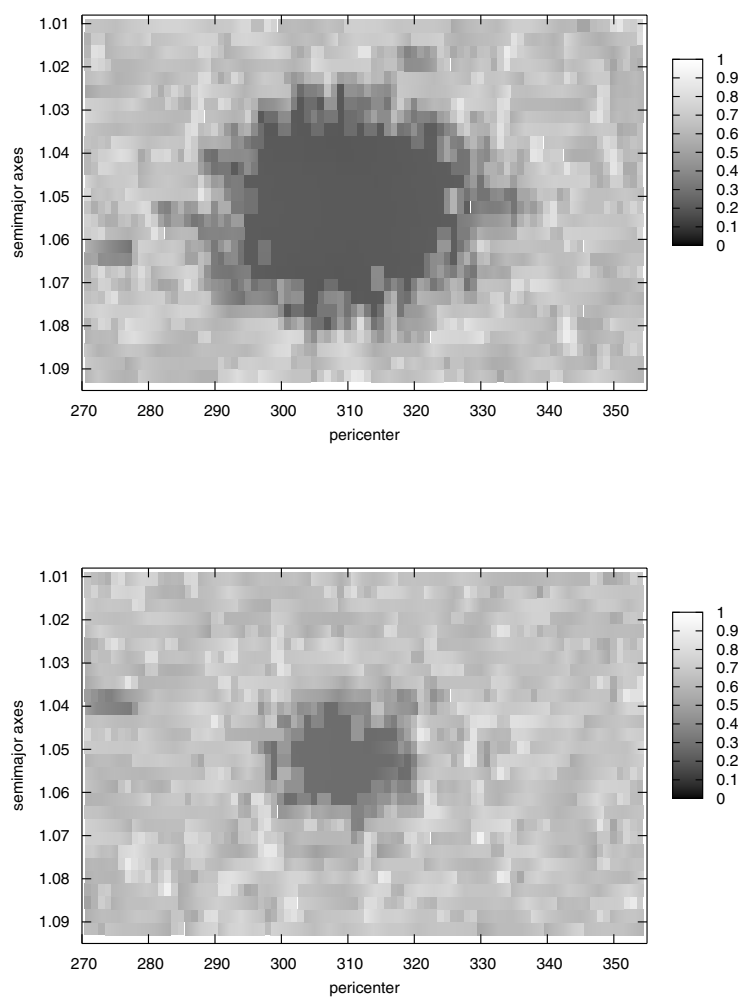

Fig. 5. Stability regions around $L_{4}$ in Models M21 and M22. The initial semimajor axis ( $y$-axis) is plotted versus the initial angular distance from the equilibrium point $L_{4}$. The dark regions around the point $L_{4}$ ( $a=1.051 \mathrm{AU}, \omega=308$ ) show that the maximum eccentricity during the integration time was always $e_{\max }<0.3$ with such initial conditions.

Table 4. Extensions of stable regions for different models with respect to the semimajor axes and the angular distance to the Langange point $L_{4}$.

\begin{tabular}{ccccccc}
\hline \hline & M21 & M22 & M31 & M32 & M21 $^{*}$ & M31 $^{*}$ \\
\hline$a(\mathrm{AU})$ & 2.68 & 2.68 & 2.93 & 2.93 & 2.68 & 2.93 \\
$e$ & 0.18 & 0.25 & 0.18 & 0.25 & 0.18 & 0.18 \\
$\delta \omega$ & $30^{\circ}$ & $14^{\circ}$ & $35^{\circ}$ & $10^{\circ}$ & $7^{\circ}$ & $22^{\circ}$ \\
$\delta a(\mathrm{AU})$ & 0.04 & 0.015 & 0.045 & 0.01 & 0.012 & 0.032 \\
\hline
\end{tabular}

the $a$ and varied $\omega^{6}$. These computations were only undertaken for the former stable regions found in models M21, M22, M31, and M32. In Fig. 8 we can see the cuts of M21 and M22 that determine the stable regions in the direction of the larger semimajor axis $a$ and with respect to the angular distance to the Lagrange point $L_{4}$. The valley of stable orbits can be approximated by an ellipse around the Langrange point, where initial conditions inside lead to stable orbits.

\section{Conclusion}

Out of the different possibilities for stable orbits of ETs in the $\mathrm{HZ}$ of EPSs, we have investigated in this paper the system HD 108874 that has two large planets: one moving in the HZ with a small eccentric orbit and another one outside with a larger eccentricity. Taking the uncertainties of the orbital parameters into account - the semimajor axis of the outer planet and its

6 The mean anomaly of the planet and the fictitious Trojan planet was always set to 0 . This choice of initial conditions ensures that for $\omega=0$ the star - the inner planet - and the Trojan form an equilateral triangle. 

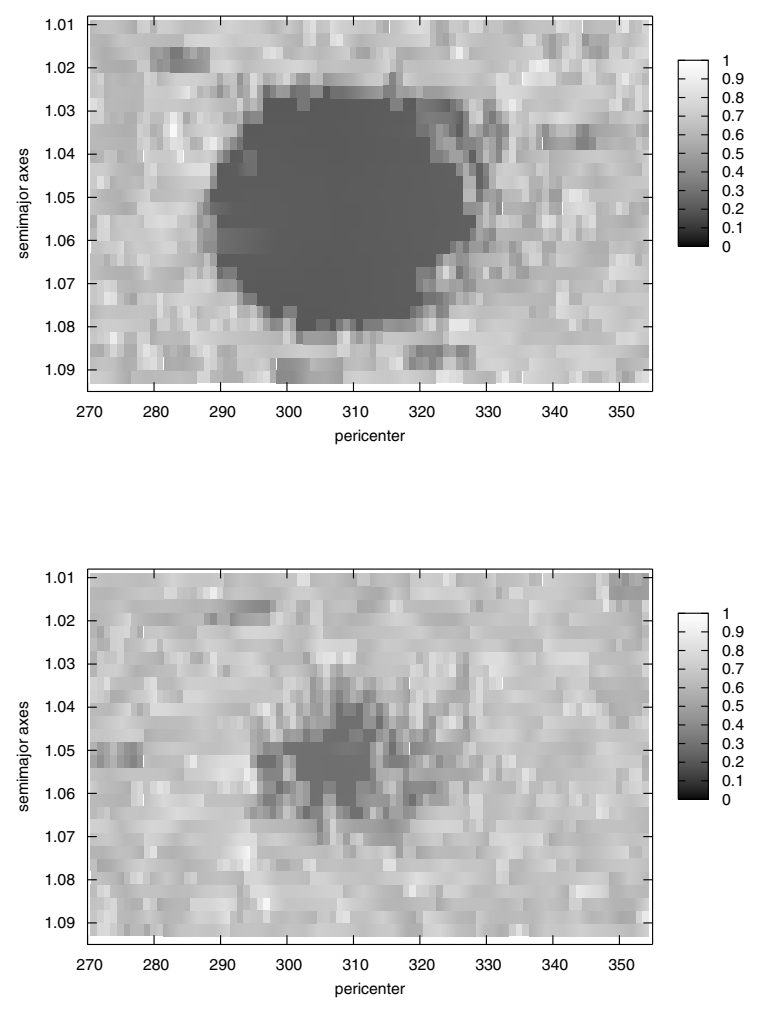

Fig. 6. Captions like in Fig. 4 but for the models M31 and M32.

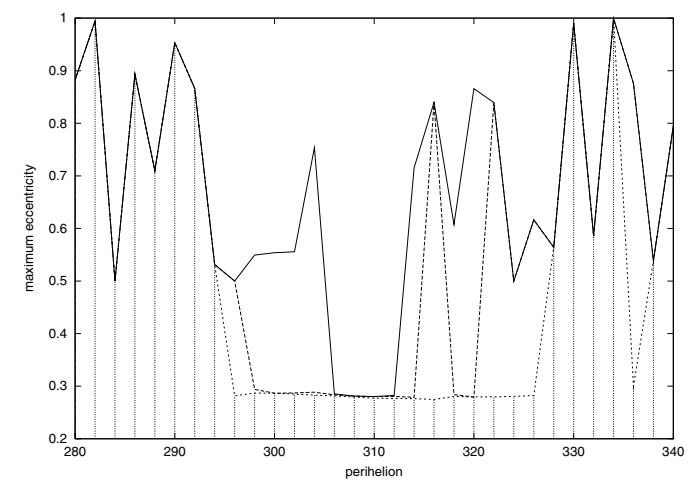

Fig. 7. Cuts with a fixed semimajor axis with changing angular distance to the Lagrange point $L_{4}$, which is located at $\omega=308^{\circ}$. The different curves show how the extension of the stable region shrinks with the integration time; full line results after $10^{7}$ years, dotted line after $10^{6}$ years, hatched region after $10^{5}$ years.

eccentricity - we made numerical simulations of orbits of the fictitious ETs of the inner planet. It turned out that the stability region for the ETs is quite small and that it disappears for most ( 5 out of 9 models) of the estimated orbital parameters. The results agree partly with former computations by Érdi \& Sándor (2005), who in their study used a different, i.e. smaller, eccentricity of the outer planet. In addition they showed how the region shrinks with the assumption of a second planet. In our study we found stable regions for ETs with orbital parameters of the two giant planets which lie well inside the error bars from the observations. Since the masses given by the observers are minimum masses for the planets involved, we checked the stability regions for higher masses, namely twice the given ones. In this case, all the Trojan orbits turn out to be unstable. With lower masses, stable regions for the model (just 50 percent heavier than the minimum masses) M21 and M31 still persist, but the
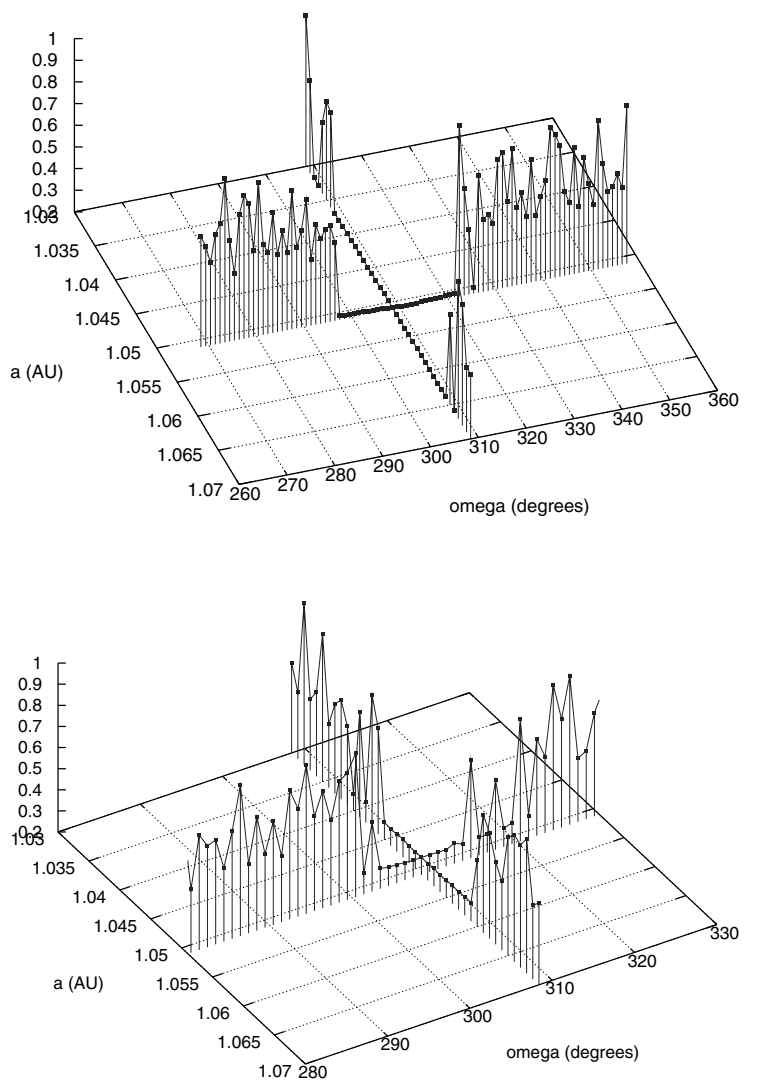

Fig. 8. Cut with a fixed semimajor axis and cut with a fixed pericenter; upper graph for model M21, lower graph for model M22. The crossing of the two lines is the Lagrange point $L_{4}$; the $z$-axis is the maximum eccentricity for the integration time of $10^{7}$ years.

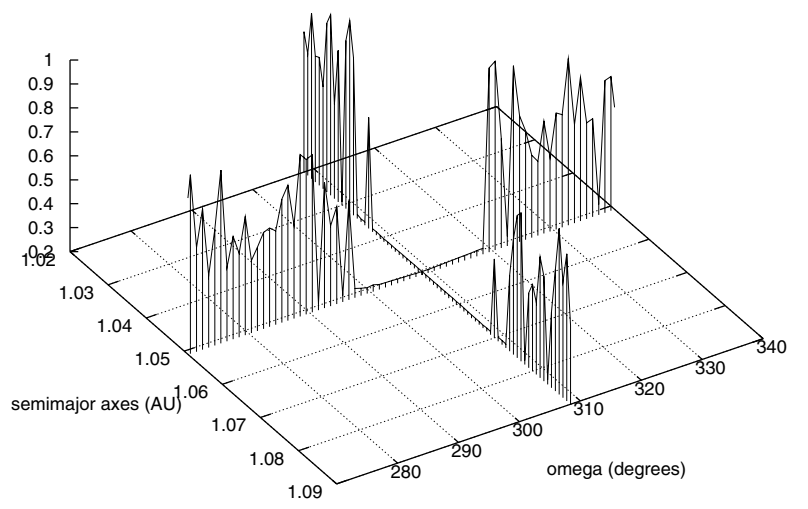

Fig. 9. Cut with a fixed semimajor axis and cut with a fixed pericenter for the model M31*. This model presents the results for more massive planets (50 percent larger) to take into account the influence of the inclination of the orbits with respect to the line of sight of the observations. The crossing of the two lines is the Lagrange point $L_{4}$; the $z$-axis is the maximum eccentricity.

extensions are somewhat smaller (see Table 4 and Fig. 9) ${ }^{7}$. We did not take the inclinations of these fictitious planets into account; but from studies of the Jupiter Trojans we know that for small eccentricities these region may be slightly larger than estimated in our study (Robutel et al. 2005). To detect a terrestrial

\footnotetext{
7 These additional computations were undertaken according to a proposition of the referee to take into consideration the possible inclination of the orbits.
} 
Trojan planet in HD 108874 via observations will be rather difficult but not impossible (Schneider 2006).

Acknowledgements. For the realization of this study we need to thank the Austrian Science Foundation (=FWF, project P16024-N05) and the ISSI Institute in Bern, which supported us in the framework of the ISSI team "Evolution of habitable planets". R.S. did this work in the framework of a Schrödinger grant of the FWF (J2619-N16); E.P.-L. wishes to acknowledge the support by the FWF Herta Firnberg grant (T122). Thanks also go to the "WissenschaftlichTechnische Zusammenarbeit Österreich-Ungarn" project A12-2004: Dynamics of extrasolar planetary Systems.

\section{References}

Asghari, N., Broeg, C., Carone, L., et al. 2004, A\&A, 426, 353

Dvorak, R., Pilat-Lohinger, E., Funk, B., \& Freistetter, F. 2003a, A\&A, 398, L1 Dvorak, R., Pilat-Lohinger, E., Funk, B., \& Freistetter, F. 2003b, A\&A, 410, L13 Dvorak, R., Pilat-Lohinger, E., Schwarz, R., \& Freistetter, F. 2004, A\&A, 426, 37

Érdi, B., \& Sándor, Zs. 2005, CMDA, 92, 113

Froeschlé, C., Lega, E., \& Gonczi, R. 1997, CMDA, 67, 41

Hanslmeier, A., \& Dvorak, R. 1984, A\&A, 132, 203
Jones, B. W., \& Sleep, P. N. 2002, A\&A, 393, 1015

Kasting, J. F., Whitmire, D. P., \& Reynolds, R. T. 1993, Icarus, 101, 108

Lammer, H., Kulikov, Yu., Penz, T., et al. 2005, in A Comparison of the Dynamical Evolution of Planetary Systems, ed. R. Dvorak, \& S. Ferraz-Mello (Springer), 273

Laughlin, G., \& Chambers, J. E. 2002, AJ, 124, 592

Lichtenegger, H. 1984, CMDA, 34, 357

Marchal, C. 1990, The Three-Body Problem (Elsevier), 49

Menou, K., \& Tabachnik, S. 2003, AJ, 583, 473

Nauenberg, M. 2002, AJ, 124, 2332

Pál, A., \& Sándor, Zs. 2003, Proceedings of the 3rd Austrian-Hungarion Workshop on Trojans and related topics, ed. F. Freistetter, R. Dvorak, \& B. Érdi, 25

Pilat-Lohinger, E., \& Dvorak, R. 2002, CMDA, 82, 143

Pilat-Lohinger, E., Funk, B., \& Dvorak, R. 2003, A\&A, 400, 1085

Robutel, P., Gabern, F., \& Jorba, A. 2005, Celest. Mech. Dyn. Astron., 92, 53

Schneider, J. 2006, private communication

Schwarz, R., Pilat-Lohinger, E., Dvorak, R., Érdi, B., \& Sándor, Zs. 2005, Astrobiol. J., 5, 579

Schwarz, R. 2005b, Ph.D. Thesis, University of Vienna, online database: http://media.obvsg.at/dissd

Vogt, S. S., Butler, P. R., Marcy, W. G., et al. 2002, AJ, 568, 352

Vogt, S. S., Butler, P. R., Marcy, W. G., et al. 2005, AJ, 632, 638 